\title{
Design of optimal laser pulses to control molecular rovibrational excitation in a heteronuclear diatomic molecule ${ }^{\#}$
}

\author{
SITANSH SHARMA ${ }^{\mathrm{a}}$, GABRIEL G BALINT-KURTI ${ }^{\mathrm{b}}$ and HARJINDER SINGH ${ }^{\mathrm{a}}$ * \\ ${ }^{a}$ Center for Computational Natural Sciences and Bioinformatics, International Institute of Information \\ Technology, Hyderabad 500 032, India \\ ${ }^{\mathrm{b}}$ Centre for Computational Chemistry, School of Chemistry, University of Bristol, Bristol BS8 1TS, UK \\ e-mail: laltu@iiit.ac.in
}

\begin{abstract}
Optimal control theory in combination with time-dependent quantum dynamics is employed to design laser pulses which can perform selective vibrational and rotational excitations in a heteronuclear diatomic system. We have applied the conjugate gradient method for the constrained optimization of a suitably designed functional incorporating the desired objectives and constraints. Laser pulses designed for several excitation processes of the $H F$ molecule were able to achieve predefined dynamical goals with almost $100 \%$ yield.
\end{abstract}

Keywords. Optimal control; conjugate gradient; heteronuclear; laser pulse design.

\section{Introduction}

State selective control of molecular dynamics by designed laser pulses has attracted attention in recent years. ${ }^{1-8}$ Selective molecular state excitations between preselected initial and final states have been successfully carried out to achieve various dynamical goals, such as bond dissociation, ${ }^{9-15}$ isotope selectivity ${ }^{16-18}$ and isomerization. ${ }^{19-21}$ Recently, in the area of quantum computing, ${ }^{22}$ use of molecular states of various systems as qubits has been tested. It has been demonstrated that using molecular states as qubits, logic gate operations can be performed with the help of specially tailored laser pulses. ${ }^{23-29}$ Within the scope of these applications, the coherent preparation of molecular states has also gained a lot of importance.

In our earlier work, we have designed optimal laser pulses which can selectively transfer population from one vibrational state to another state. ${ }^{8}$ In the present work, we demonstrate the application of optimal control theory ${ }^{30,31}$ to design infrared laser pulses for selective rovibrational excitations in a heteronuclear diatomic molecule $H F$. In order to restrict the frequency and amplitude structures of the pulses, the conjugate gradient method in combination with frequency filtering and

\footnotetext{
\#Dedicated to Prof. N Sathyamurthy on his 60th birthday

*For correspondence
}

amplitude projection technique as suggested by BalintKurti et al. ${ }^{32}$, has been employed. The conjugate gradient method has recently been applied within the context of the optimal control theory to several vibrational and rotational excitations. ${ }^{33-38}$

Our aim is to design a laser pulse which can selectively and completely transfer population from a given initial quantum state to the desired target state. The interaction of the laser light with the molecule is treated within the dipole approximation, ${ }^{39,40}$ which means that only the interaction of the electric field vector of the radiation with the geometry-dependent electric dipole moment of the molecule has been considered. Laser pulses have been designed for following three vibrational and rotational excitations using full three-dimensional time-dependent quantum dynamics

$$
\begin{aligned}
& H F(v=0, j=0) \rightarrow H F(v=1, j=1), \\
& H F(v=1, j=1) \rightarrow H F(v=2, j=0), \\
& H F(v=0, j=0) \rightarrow H F(v=1, j=0) .
\end{aligned}
$$

The first two excitation processes are dipole allowed transitions while the last one is a dipole forbidden transition.

Section 2 briefly outlines the optimal control theory used, and the use of the conjugate gradient method for the optimization of the laser pulse shape. Results and discussion are presented in Section 3. Finally, in Section 4 we present a conclusion. 


\section{Theory}

\subsection{Optimal control theory}

Our aim is to optimize the electric field of a laser pulse so as to transform the nuclear wave function of a system from an initial molecular state, $\psi(t=0)$, to a desired target molecular state, $\phi$, at a fixed time $t=T$. Time-dependent quantum mechanics is used to describe the motion of the system interacting with the timedependent electric field of the laser pulse along with an optimization algorithm. In order to design an optimal laser pulse, we define a grand objective functional, $J$, of the following form ${ }^{32}$

$$
\begin{aligned}
J[\varepsilon]= & |\langle\psi(T) \mid \phi\rangle|^{2}-\alpha_{0} \int_{0}^{T}|\varepsilon(t)|^{2} d t \\
& -2 \operatorname{Re}\left[\int_{0}^{T}\left\langle\chi(t)\left|\frac{\partial}{\partial t}+i \hat{\mathrm{H}}(t)\right| \psi(t)\right\rangle d t\right] .
\end{aligned}
$$

The functional has three terms: (a) an objective term which is the overlap of the field propagated initial wavefunction with the target wavefunction, $|\langle\psi(T) \mid \phi\rangle|^{2}$, where the quantity $\psi(T)$ is the initial wavefunction propagated to time $T$ under the action of the laser field $\varepsilon(t)$ and $\phi$ is the target molecular state to be reached at the final time $T$ (we refer this term as the transition probability), (b) a penalty term, $\alpha_{0} \int_{0}^{T}|\varepsilon(t)|^{2}$, for the fluence which restricts the magnitude of the electric field; $\alpha_{0}$ is a constant positive weighting parameter that specifies the weight of the fluence penalty term in the functional, and (c) a dynamical constraint that the time-dependent Schrödinger equation must be obeyed by the wavefunction $\psi(t)$ at all time, where $\chi(t)$ is a Lagrange multiplier introduced to assure satisfaction of the time-dependent Schrödinger equation. $\hat{\mathrm{H}}(t)$ is the overall Hamiltonian of the system which includes laser-matter interaction term.

The time evolution of the molecular wavefunction $\psi(t)$ under the influence of external field $\varepsilon(t)$ is governed by the time dependent Schrödinger equation which may be written in the form:

$$
i \frac{\partial}{\partial t} \psi(t)=\hat{\mathrm{H}}(t) \psi(t)=\left[\hat{\mathrm{H}}_{0}+\hat{\mathrm{H}}_{1}(t)\right] \psi(t),
$$

where $\hat{\mathrm{H}}_{0}$ is the molecular Hamiltonian in the absence of laser field and $\hat{\mathrm{H}}_{1}(t)$ is the interaction Hamiltonian.
Using the full three-dimensional system, the molecular Hamiltonian can be written as

$$
\begin{aligned}
\hat{\mathrm{H}}_{0}= & -\frac{1}{2 m r^{2}} \frac{\partial}{\partial r}\left(r^{2} \frac{\partial}{\partial r}\right) \\
& -\frac{1}{2 m r^{2}} \frac{1}{\sin \theta} \frac{\partial}{\partial \theta}\left(\sin \theta \frac{\partial}{\partial \theta}\right) \\
& -\frac{1}{2 m r^{2} \sin ^{2} \theta}\left(\frac{\partial^{2}}{\partial \phi^{2}}\right)+V(r),
\end{aligned}
$$

where $V(r)$ is the potential energy function, $r$ is the internuclear distance and $\theta$ and $\phi$ are the angles that describe the orientation of the molecule.

We assume that the electric field is pointed in the positive $z$ direction. Within the semiclassical dipole approximation, the interaction Hamiltonian can be written as:

$$
\hat{\mathrm{H}}_{1}(t)=-\mu(r) \varepsilon(t) \cos \theta,
$$

the dipole moment, $\mu(r)$, is given as,

$$
\mu(r)=\mu_{0} r e^{-\sigma r^{4}},
$$

where $\mu_{0}=0.4535$ and $\sigma=0.0064$ in a. u. ${ }^{41}$

Variation of $J$ (see Eq. 2) with small independent changes in the wavefunction, $\psi(t)$, the Lagrange multiplier, $\chi(t)$, and the electric field, $\varepsilon(t)$, leads to the following set of pulse design equations for the optimal control of nuclear motion.

$$
\begin{aligned}
i \frac{\partial \psi(t)}{\partial t} & =\hat{\mathrm{H}} \psi(t), \quad \psi(0)=\phi, \\
i \frac{\partial \chi(t)}{\partial t} & =\hat{\mathrm{H}} \chi(t), \quad \chi(T)=\langle\phi \mid \psi(T)\rangle \phi, \\
\alpha_{0} \varepsilon(t) & =\operatorname{Im}\left(\left\langle\chi(t)\left|\frac{\partial \hat{\mathrm{H}}(t)}{\partial t}\right| \psi(t)\right\rangle\right) .
\end{aligned}
$$

In general, these coupled differential equations should be solved iteratively. In the present work, in order to maximize the objective functional, $J$, we calculate the variation of functional with respect to a small change in electric field component of the laser pulse using the conjugate-gradient method described below.

\subsection{Optimization algorithm}

In order to design the laser field, $\varepsilon(t)$, which can selectively transfer population between two preselected states, we compute the derivative of the objective functional $J$ with respect to the electric field of the laser pulse at time $t .{ }^{33}$ The objective functional may then be maximized using the conjugate-gradient method. ${ }^{42}$ The laser field, $\varepsilon(t)$, is defined as a product of a Gaussian envelope function $s(t)$, which is practically zero at $t=0$ 
and at end of the pulse, $t=T$, and a part $\varepsilon_{0}(t)$ which will be optimized to achieve the desired objective, i. e.,

$$
\varepsilon(t)=\varepsilon_{0}(t) . s(t),
$$

here, $s(t)=\exp \left[\frac{-(t-T / 2)^{2}}{(T / 4)^{2}}\right]$, which is preserved throughout the optimization to ensure an experimentally feasible profile for the laser pulse.

The gradient of $J$ with respect to $\varepsilon_{0}(t)$ at time $t$ after $k$ iterations of the optimization cycle is written as

$$
\begin{aligned}
g^{k}(t)= & \frac{\partial J^{k}}{\partial \varepsilon_{0}^{k}(t)}=-2 s(t) \\
& \times\left[\alpha_{0} \varepsilon^{k}(t)-\operatorname{Im}\left\langle\chi(t)\left|\frac{\partial \hat{\mathrm{H}}(t)}{\partial \varepsilon^{k}(t)}\right| \psi(t)\right\rangle\right],
\end{aligned}
$$

The Polak-Ribiere-Polyak search direction is defined using the gradient given in Eq. 11 as

$$
d^{k}\left(t_{i}\right)=g^{k}\left(t_{i}\right)+\frac{\sum_{i} g^{k}\left(t_{i}\right)^{T}\left(g^{k}\left(t_{i}\right)-g^{k-1}\left(t_{i}\right)\right)}{\sum_{i} g^{k-1}\left(t_{i}\right)^{T} g^{k-1}\left(t_{i}\right)} d^{k-1}\left(t_{i}\right),
$$

where $k=2,3, \ldots$, and $d^{1}\left(t_{i}\right)=g^{1}\left(t_{i}\right)$. In order to restrict the algorithm from sampling $\varepsilon(t)$ values outside of the predefined electric-field amplitude range during the line search, the direction $d_{k}(t)$ is projected to obtain $d_{p}^{k}(t) .{ }^{43}$ The projected search direction, $d_{p}^{k}(t)$, is first transformed from a time-dependent to a frequencydependent quantity using the Fast Fourier transform method, ${ }^{44}$ and this function is then filtered using a $20^{\text {th }}$ order Butterworth bandpass filter. ${ }^{45}$ The resulting quantity is then inverse Fourier transformed to yield a timevarying electric field which excludes very high and low frequency components. The updated time-dependent electric field can be given as

$$
\varepsilon^{k+1}(t)=\varepsilon^{k}(t)+\lambda s(t) d_{p}^{k}(t),
$$

where $\lambda$ is determined by a line search.

\section{Results and discussion}

Our goal is to design a laser pulse having simple time and frequency structure which can selectively transfer population from a given initial rovibrational state to a desired target state. We have considered the $H F$ molecule initially in its ground state as the model system. The atomic interactions for the $H F$ model system is described using Morse potential,

$$
V(r)=D_{e}\left[1-e^{-\beta\left(r-r_{e}\right)}\right]^{2},
$$

where, $D_{e}=0.2250073497$ Hartree, $r_{e}=1.7329 \mathrm{Bohr}$ and $\beta=1.1741$ a.u. ${ }^{42}$ For a full three-dimensional treatment, the nuclear wavefunction is represented on a two-dimensional grid of $r$ and $\theta$, where $r$ is the internuclear distance and $\theta$ is the polar angle between the field polarization direction and the molecular axis. The coordinate grid in $r$ consists of evenly spaced grid points while the $\theta$ grid points are the Gauss-Legendre quadrature points. ${ }^{46}$ The nuclear wavefunction used is represented on a spatial grid spanning an $\mathrm{H}-\mathrm{F}$ bond distance from 0.34658 to $5.1987 \mathrm{Bohr}$ in 64 steps and 16 angular points. The Fourier grid Hamiltonian method is employed to compute the radial part of the initial nuclear wavefunction. ${ }^{47}$ The time evolution of the wavefunction under the action of the electric field of the laser pulse is performed by solving time-dependent Schrödinger equation using the split operator method. ${ }^{48}$ The action of the radial dependent part of the kinetic energy operator on the initial wavefunction is evaluated using the Fast Fourier Transform method ${ }^{49,50}$ and the action of the $\theta$ dependent part of the kinetic energy operator is evaluated using the discrete variable representation method. ${ }^{46,51}$

\section{$3.1 H F(v=0, j=0) \rightarrow H F(v=1, j=1)$ excitation (i): $(\Delta v=+1, \Delta j=+1)$}

The excitation $(i)$ is a dipole allowed transition in which both the vibrational as well as rotational quantum numbers increase by one. The initial form of the laser field is defined as

$$
\varepsilon(t)=0.006 \sin \left(\omega_{v=0, j=0 \rightarrow v=1, j=1} t\right) s(t) .
$$

In this case, the system is propagated for 50,000 a.u. (1.21 ps) of time. The pulse length is divided into 65536 time steps. The electric field of the optimized laser pulse has a maximum amplitude of 0.00542 a.u. $\left(2.79 \times 10^{9}\right.$ $\mathrm{Vm}^{-1}$ ) and is shown in plot $a 1$ of figure 1 . The frequency spectrum (plot a2) shows the main contribution from the $\omega_{v=0, j=0 \rightarrow v=1, j=1}$ transition frequency. The time variation of the population of the different quantum states of $H F$, which are significantly populated during the action of laser pulse, is shown in plot a3. The intermediate states $(v=0, j=1),(v=0, j=2)$, $(v=1, j=0)$ and $(v=1, j=2)$ are populated to a very small extent during the dynamics. At the end of the pulse duration, nearly $100 \%$ of the population is transferred to the desired molecular state.

3.2 $H F(v=1, j=1) \rightarrow H F(v=2, j=0)$ excitation (ii): $(\Delta v=+1, \Delta j=-1)$

The transition (ii) is also a dipole allowed transition wherein the vibrational quantum number is 

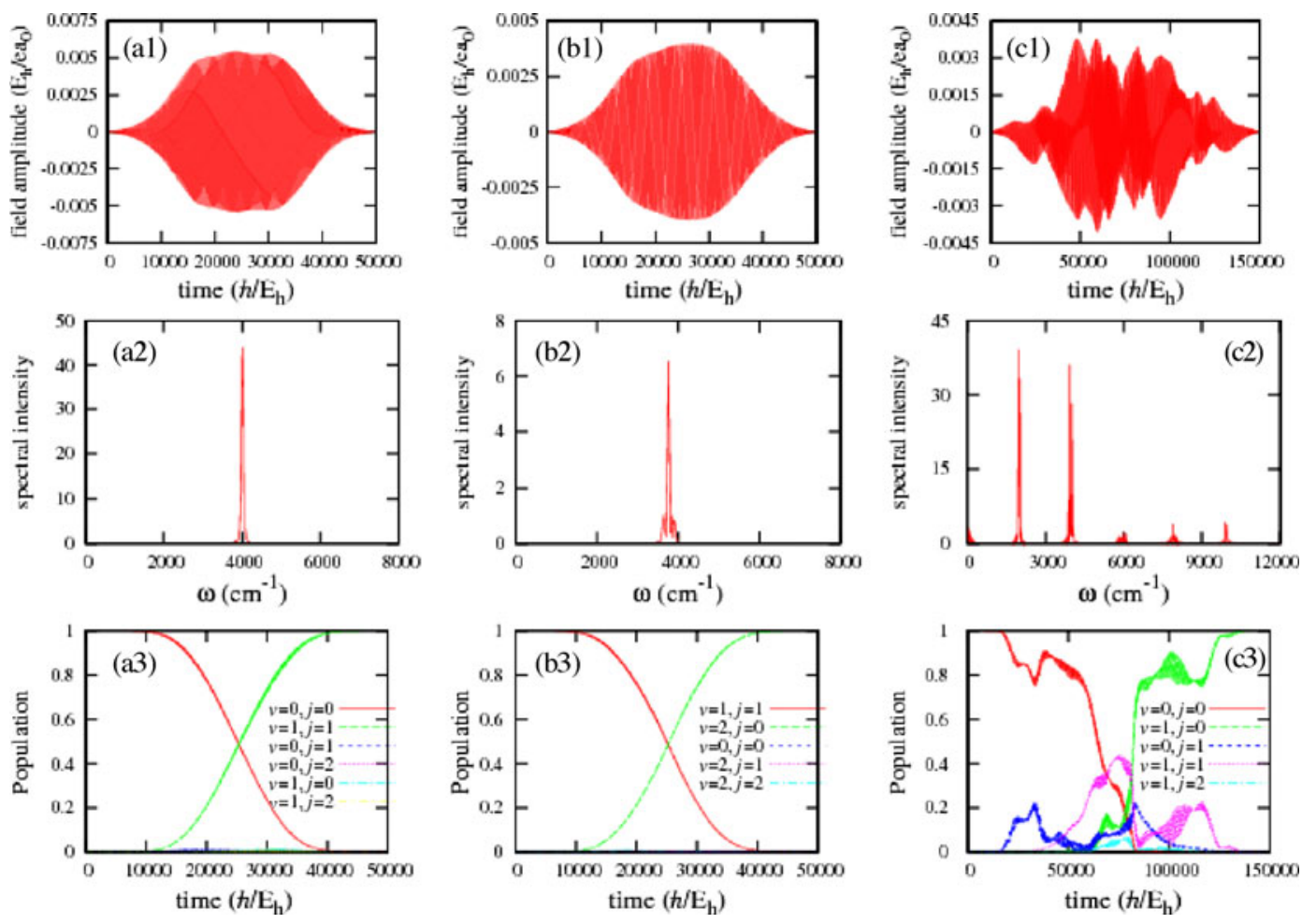

Figure 1. Results for selective vibrational and rotational excitations: Optimized field as a function of time (a1, b1, c1), frequency spectrum of the optimized field $(\mathbf{a} 2, \mathbf{b 2}, \mathbf{c 2})$, quantum state population as a function of time $(\mathbf{a 3}, \mathbf{b 3}, \mathbf{c 3})$ for $v=0, j=0 \rightarrow v=1$, $j=1(\mathbf{a 1}-\mathbf{a 3}), v=1, j=1 \rightarrow v=2, j=0(\mathbf{b 1 - b 3})$ and $v=0, j=0 \rightarrow v=1, j=0(\mathbf{c 1}-\mathbf{c} 3)$ transitions in $H F$ molecule; $\alpha_{0}$ is set as 0.1 .

increased by one while the rotational quantum number is decreased by one. For this transition, a pulse of duration 50,000 a.u. (1.21 ps) is used and the pulse length is divided into 65536 time steps. The initial laser field has the form

$$
\varepsilon(t)=0.006 \sin \left(\omega_{v=1, j=1 \rightarrow v=2, j=0} t\right) s(t) .
$$

Plots $b 1$ and $b 2$ of figure 1 show the electric field and the frequency spectrum of the optimized pulse. The field has a maximum amplitude of 0.004 a.u. $\left(2.04 \times 10^{9}\right.$ $\mathrm{Vm}^{-1}$ ). The frequency plot $b 2$ shows that the optimization process has introduced small secondary frequency peaks along with the major peak around the transition frequency from $\omega_{v=1, j=1 \rightarrow v=2, j=0}$. Insights into the mechanism of the population transfer during the excitation process can be gained by plotting the population of various quantum states of $H F$ as a function of time, as shown in plot $b 3$. Again there is very little excitation of many intermediate quantum states.

$$
\text { 3.3 } H F(v=0, j=0) \rightarrow H F(v=1, j=0) \text { excitation }
$$$$
\text { (iii): }(\Delta v=+1, \Delta j=0)
$$

The excitation (iii) is a dipole forbidden meaning transition, that it would not take place if the vibrational and rotational motions were uncoupled such as, the vibrational motion being purely harmonic and the dipole moment being a linear function of the internuclear separation. These are the normal assumptions used in discussing infrared spectroscopy. In order to achieve this transition, the initial laser field is chosen as

$$
\varepsilon(t)=0.01 \sin \left(\frac{\omega_{v=0, j=0 \rightarrow v=1, j=0}}{2} t\right) s(t) .
$$

In this case, a pulse of relatively longer duration, viz. 150,000 a.u. (3.63 ps) has been chosen. The pulse length is divided into 131072 time steps. The converged optimized field as a function of time is shown in plot cl of figure 1. The maximum amplitude is 0.00406 a.u. $\left(2.09 \times 10^{9} \mathrm{Vm}^{-1}\right)$. The frequency spectrum is shown in plot $c 2$. The plot shows that the main contributions come from around $\left(\omega_{v=0, j=0 \rightarrow v=1, j=0}\right) / 2$ (initial frequency) and $\omega_{v=0, j=1 \rightarrow v=1, j=0}$, along with small contributions from higher order harmonics. The sharp variation of field amplitude in plot $c l$ results in secondary frequency components in the frequency spectrum of the field (c2). The population of the various quantum states of $H F$ which are significantly populated during the excitation process are plotted as a function of time in $c 3$. The plot shows the complex nature of the population transfer. Several rotational states are populated as 
Table 1. Summarized results for the three examined excitations. ' $P$ ' refers to transition probability; ' $J$ ' refers to cost functional value; ' $\varepsilon_{\text {peak }}$ ' refers to maximum amplitude of the optimized laser pulse.

\begin{tabular}{lccc}
\hline Transition & $P$ & $J$ & $\varepsilon_{\text {peak }}$ (a.u.) \\
\hline$H F(v=0, j=0) \rightarrow H F(v=1, j=1)$ & 0.99926 & 0.96796 & $5.423 \times 10^{-3}$ \\
$H F(v=1, j=1) \rightarrow H F(v=2, j=0)$ & 0.99981 & 0.98394 & $3.966 \times 10^{-3}$ \\
$H F(v=0, j=0) \rightarrow H F(v=1, j=0)$ & 0.99980 & 0.97507 & $4.063 \times 10^{-3}$ \\
\hline
\end{tabular}

the system evolves in time. Eventually, all these intermediate states are depopulated and whole of this population is transferred to the target state. Table 1 shows that at the end of the pulse duration, more than $99 \%$ of population is transferred to the desired quantum state $(v=1, j=0)$.

The complex nature of the quantum state population as a function of time as shown in figure $1(c 3)$, and the fact that a much longer overall time was needed to achieve this excitation process, both attributed to the dipole forbidden nature of the process.

\section{Conclusion}

We have applied a frequency filtering technique along with the conjugate gradient method within the frame work of OCT to design laser pulses which can selectively perform controlled vibrational and rotational quantum state excitation in a heteronuclear diatomic molecule. The design techniques have been applied to achieve selective population transfer for several rovibrational excitation processes, some of which are not dipole allowed for a one-photon process. Results are discussed here for three rovibrational excitation processes.

For all the studied transitions, the designed laser pulses are able to transfer almost $100 \%$ population to the desired target molecular state. Laser pulses designed for dipole allowed transitions are much simpler in time and frequency structure compared to the single dipole forbidden transition studied here. The complex time variation of the electric field component of the laser pulse for dipole forbidden transition leads to complex frequency spectrum, which in turn leads to the complex mechanism of population transfer under the action of the laser field.

\section{Acknowledgements}

SS thanks the Council of Scientific and Industrial Research (CSIR), New Delhi for a senior research fellowship. HS thanks the Department of Science and Technology, India (DST), for support.

\section{References}

1. Bloembergen N and Zewail A H 1984 J. Phys. Chem. 88 5459

2. Cantrell C D (ed.) 1986 Multi-photon excitation and dissociation of polyatomic molecule (Berlin: Springer)

3. Bandrauk A D (ed.) 1988 Atomic and molecular processes with short intense laser pulses (New York: Plenum)

4. Brumer P and Shapiro M 1992 Annu. Rev. Phys. Chem. 43257

5. Sharma S, Sharma P, Singh H and Balint-Kurti G G 2009 J. Mol. Model 15623

6. Singh H, Sharma S, Kumar P, Harvey J and Balint-Kurti G G 2008 Lect. Notes Comput. Sci. 5102387

7. Sharma S, Singh H and Balint-Kurti G G 2010 J. Chem. Phys. 132064108

8. Sharma S, Sharma P and Singh H 2007 J. Chem. Sci. 119433

9. Shi S and Rabitz H 1991 Comp. Phys. Commun. 6371

10. Jakubetz W, Kades E and Manz J 1993 J. Phys. Chem. 9712609

11. Korolkov M V, Manz J and Paramonov G K 1997 Chem. Phys. S341

12. Andrianov I V and Paramonov G K 1999 Phys. Rev. A 592134

13. Zhao Y and Kühn O 1999 Chem. Phys. Lett. 3027

14. Levis R J, Menkir G M and Rabitz H 2001 Science 292 709

15. Brixner T and Gerber G 2003 Chem. Phys. Chem. 4418

16. Ohtsuki Y and Fujimura Y 2007 Chem. Phys. 338285

17. Lindinger A, Lupulescu C, Plewicki M, Vetter F, Merli A, Weber S M and L. Wöste 2004 Phys. Rev. Lett. 93 033001

18. Kurosaki Y, Yokoyama K and Yokoyama A $2009 \mathrm{~J}$. Chem. Phys. 131144305

19. Combariza J E, Manz J and Paramonov G K 1991 Faraday Discuss. Chem. Soc. 91358

20. Großmann F, Feng L, Schmidt G, Kunert $\mathrm{T}$ and R. Schmidt 2002 Europhys. Lett. 60201

21. Artamonov M, Ho T -S and Rabitz H 2006 Chem. Phys. 328147

22. Nielsen M A and Chuang I L 2006 Quantum computation and quantum information (London: Cambridge University Press)

23. Vala J, Amitay Z, Zhang B, Leone S R and Kosloff R 2002 Phys. Rev. A 66062316

24. Ohtsuki Y 2005 Chem. Phys. Lett. 404126

25. de Vivie-Riedle R and Troppmann U 2007 Chem. Rev. 1075082 
26. Babikov D 2004 J. Chem. Phys. 1217577

27. Shioya K, Mishima K, and Yamashita K 2007 Mol. Phys. 1051283

28. Tsubouchi M and Momose T 2008 Phys. Rev. A 77 052326

29. Zaari R R and Brown A 2010 J. Chem. Phys. 132014307

30. Rice S A and Zhao M 2000 Optical control of molecular dynamics (New York: Wiley Interscience)

31. Shapiro M and Brumer P 2003 Principles of the quantum control of molecular processes (Canada: John Wiley and Sons)

32. Balint-Kurti G G, Zou S and Brown A 2008 Adv. Chem. Phys. 13843

33. Balint-Kurti G G, Manby F R, Ren Q, Artamonov M, Ho T -S and Rabitz H 2005 J. Chem. Phys. 122084110

34. Ren Q, Balint-Kurti G G, Manby F R, Artamonov M, Ho T -S and Rabitz H 2006 J. Chem. Phys. 124 014111

35. Ren Q, Balint-Kurti G G, Manby F R, Artamonov M, Ho T -S and Rabitz H 2006 J. Chem. Phys. 125 021104

36. Hermann T, Ren Q, Balint-Kurti G G and Manby F R 2007 J. Chem. Phys. 126224309

37. Zou S, Balint-Kurti G G and Manby F R 2007 J. Chem. Phys. 127044107
38. Ren Q and Balint-Kurti G G 2009 J. Phys. Chem. A 113 14255

39. Shi S and Rabitz H 1990 J. Chem. Phys. 92364

40. Sundermann K and de Vivie-Riedle R 1999 J. Chem. Phys. 1101896

41. Lin J T and Jiang T F 1999 J. Phys. B: At. Mol. Opt. Phys. 324001

42. Press W H, Teukolsky S A, Vetterling W T and Flannery B P 2000 Numerical recipes (Cambridge: Cambridge University Press)

43. Birgin E G, Martinez J M and Raydan M 2000 SIAM J. Optim. 101196

44. Kosloff R 1988 J. Phys. Chem. 922087

45. Rabiner L R and Rader C M (eds.) 1972 Digital signal processing: IEEE press selected reprint series (New York: IREEE press)

46. Offer A R and Balint-Kurti G G 1994 J. Chem. Phys. 10110416

47. Marston C C and Balint-Kurti G G 1989 J. Chem. Phys. 913571

48. Feit M D and Fleck J A Jr 1983 J. Chem. Phys. 78301

49. Kosloff D and Kosloff R 1983 J. Comp. Phys. 5235

50. Kosloff R 1988 J. Phys. Chem. 922087

51. Light J C, Hamilton I P and Lill V J 1985 J. Chem. Phys. 821400 